\title{
Phase Diagram of the 2-Leg Heisenberg Ladder with Alternating Dimerization
}

\author{
M.A. Martín-Delgado ${ }^{1}$, J. Dukelsky ${ }^{2}$ and G. Sierra ${ }^{3}$ \\ ${ }^{1}$ Departamento de Física Teórica I, Universidad Complutense. Madrid, Spain. \\ ${ }^{2}$ Instituto de Estructura de la Materia, C.S.I.C.,Madrid, Spain. \\ ${ }^{3}$ Instituto de Matemáticas y Física Fundamental, C.S.I.C., Madrid, Spain.
}

\begin{abstract}
Using the Lanczos method we determine the phase diagram of the 2-leg AFHeisenberg ladder with alternating dimerization. It consists of a resonating valence bond phase and a dimer phase separated by a critical line. Our results are in good agreement with previous conjectures obtained with the non linear sigma model.
\end{abstract}

PACS number: 75.10.Jm

Typeset using REVTEX 
In the last few years, Heisenberg spin ladders have attracted considerable attention [1] due to possible connections to materials exhibiting high $\mathrm{T}_{c}$ superconductivity. Theoretical studies [2] hint at the possibility that even-legged ladders become superconductors when doped with charge carriers. There is some experimental support for this possibility as $\mathrm{Sr}_{0.4} \mathrm{Ca}_{13.6} \mathrm{Cu}_{24} \mathrm{O}_{41.84}$, a material with spin- $\frac{1}{2}$ chains and 2-chain ladders, was shown to superconduct at $12 \mathrm{~K}$ and $3 \mathrm{GPa}[3]$.

Spin ladders are also interesting theoretically because of their unexpected behavior when viewed as interpolating constructions between the spin- $\frac{1}{2} 1 \mathrm{D}$ antiferromagnetic Heisenberg chain and their 2D square analog. The latter is ordered at low temperatures [四], while the former exhibits spin-spin correlations with a power-law decay along the 1D chain. Yet the transition between these limits by forming spin- $\frac{1}{2}$ ladders with increasing numbers of legs is not smooth: ladders with even numbers of legs have a finite gap to the lowest triplet state and an exponential decay of spin-spin correlations, while odd-leg ladders have gapless excitations and a power-law fall off of spin-spin correlations. These issues and examples of materials exhibiting these properties are discussed in several recent reviews [1] (and references therein).

With the hope of gaining a deeper insight into such systems, a variety of techniques have been employed, such as exact diagonalizations with the Lanczos algorithm [5], quantum Monte Carlo simulations [5] [6], and density matrix renormalization group methods [7] [2]. Lanczos calculations, while limited to small size $L$ of the lattice, play an important role in testing approximate schemes [8] and also in evaluating dynamic quantities that are difficult to treat in other approaches.

In this letter we present a Lanczos [9] [10] study of the 2-leg AF-Heisenberg ladder with alternating dimerization. By alternating dimerization we mean that the exchange coupling constants along the legs are not uniform in strength, but change according to a definite pattern shown in Fig.1a. The choice of this pattern among many other possibilities is not arbitrary, as was shown in reference [1]] where a great variety of quantum critical phases were conjectured and classified using the Haldane mapping of spin systems into the non linear 
sigma model. The columnar dimerization of the two legged ladder has been considered in reference [12], where it was shown that a critical line emerges for ferromagnetic inter-leg couplings and antiferromagnetic intra-leg ones.

Here we present Lanczos results for the singlet-triplet gap in $2 \times L$ systems with $L=4$, $6,8,10$ and 12 . This in turn allow us to estimate the critical curve in the space of couplings of the dimerized ladder which, as will be shown below, is two dimensional. We also compare the numerical results with the analytical predictions based on perturbation theory and on the non linear sigma model [11].

To be more precise, let us start with the Hamiltonian for the spin- $\frac{1}{2}$ Heisenberg spin ladder consisting of two coupled chains with alternating couplings given by,

$$
H=\sum_{a=1}^{2} \sum_{n=1}^{L} J\left(1+(-1)^{a+1} \gamma\right) \vec{S}_{a}(n) \cdot \vec{S}_{a}(n+1)+J^{\prime} \sum_{n=1}^{L} \vec{S}_{1}(n) \cdot \vec{S}_{2}(n),
$$

where $\vec{S}_{a}(n)$ denotes the quantum spin-1/2 at site $n$ in the leg $a=1,2$ of the ladder, $J$ and $J^{\prime}$ are coupling constants along the legs and the rungs respectively, and $\gamma$ is the dimerization parameter. In order to keep the system always in the antiferromagnetic regime, we restrict the range of variation as $|\gamma| \leq 1$ (see figure 1a)). We use periodic boundary conditions along the legs of the ladder.

In the strong coupling limit $\left(J^{\prime} / J \gg 1\right)$ and in the absence of staggering $(\gamma=0)$, the spins across each rung form a singlet and the ground state wave function is the coherent superposition of these singlets [5]. The ground state thus has $S=0$ and an energy gap proportional to $J^{\prime}$, with perturbative corrections of relative size $J / J^{\prime}$. These perturbative expansions can be extended to the case of non-vanishing dimerization $\gamma \neq 0$. From second order perturbation theory we obtain for the gap,

$$
\Delta\left(\gamma, J^{\prime} / J\right)=J^{\prime}-J+\left(\frac{1}{2}-2 \gamma^{2}\right) \frac{J^{2}}{J^{\prime}}
$$

Playing with the two coupling constants present in the problem, we can tune for instance the dimerization in order to have a vanishing spin gap $\Delta\left(\gamma_{c},\left(J^{\prime} / J\right)_{c}\right)=0$. This yields the existence of a critical curve for gapless excitations which in this approximation reads as follows, 


$$
\left(J^{\prime} / J\right)_{c}=\frac{1}{2}\left(1+\sqrt{8 \gamma_{c}^{2}-1}\right),
$$

A more elegant and powerful method was used in [11] to determine the existence of critical curves for any spin $S$ and number of legs $n_{l}$ in the two-dimensional plane of coupling constants $\gamma$ and $J^{\prime} / J$. There we employed the well-known Haldane mapping [14] of a AF-Heisenberg model to the NLSM (Non-Linear Sigma Model). In this approximation, the existence of gapless excitations is detected through the condition that the $\theta$-parameter associated to the topological term of the NLSM is an odd multiple of $\pi$. In the case of a $n_{l}=2$ ladder with spin- $1 / 2$ degrees of freedom we found,

$$
\theta=2 \pi\left(1-\frac{\gamma}{1+J^{\prime} / 2 J}\right),
$$

The criticality condition $\theta=\pi$ leads in this framework to the existense of a critical curve given by,

$$
\gamma_{c}=\frac{1}{2}\left(1+J_{c}^{\prime} / 2 J_{c}\right),
$$

which is a straight line starting from $J^{\prime} / J=2$ at $\gamma=1$ and ending at $J^{\prime} / J=0$ and $\gamma=1 / 2$. The NLSM has to be taken as a guide to the physics of the problem but in general it is not quantitatively correct. Indeed at $J^{\prime} / J=0$ the critical curve has to end up at the point $\gamma=0$, corresponding to two uncoupled uniform chains. Thus the point $\left(J^{\prime} / J=0, \gamma=1 / 2\right)$ is certainly non critical. On the other hand, the point $\left(\gamma=1, J^{\prime} / J=2\right)$ corresponds to the exact solution as shown in reference [11]. The reason for this is essentially geometrical as shown in figure 1c. At $\gamma=1$ the 2-leg ladder becomes effectively a single spin chain with the shape of a snake. If moreover $J^{\prime} / J$ equals 2 this chain becomes uniform and consequently is gapless. Away from this ratio there appears a standard dimerization gap [13].

These strong-coupling arguments together with the weak coupling arguments given in reference [11] suggest the existence of a critical curve connecting the points $\left(J^{\prime} / J, \gamma\right)=(2,1)$ and $(0,0)$.

However, despite all this mounting evidence in favor of the existence of a critical curve in 
the two-dimensional space of coupling constants, it is apparent that a direct determination of this phase diagram must be reported.

Here we carry out a Lanczos diagonalization on finite $2 \times L$-site lattices in order to compute the spin gap $\Delta_{L}\left(\gamma, J^{\prime} / J\right)$ for a range of values of the coupling constants given by $0 \leq J^{\prime} / J \leq 2$ and $0 \leq \gamma \leq 1$

In Fig.2 we show a 3D-plot of the surface of gaps $\Delta\left(\gamma, J^{\prime} / J\right)_{L}$ for $L=10$ where we can inequivocally appreciate a steep valley signaling the existence of the critical curve we are searching for.

Next we perform a finite size analysis to determine the critical and non-critical regions in the space of coupling constants.

In a gapped regime the spin gap has the scaling behaviour [5]

$$
\Delta_{L}-\Delta_{\infty}=C \frac{e^{-L / \xi}}{L}
$$

where $\xi$ is the correlation length. If $\xi$ is larger than the corresponding lattice size one can actually get a better fit of the data with the formula

$$
\Delta_{L}-\Delta_{\infty}=\frac{C}{L}
$$

where $\Delta_{\infty}$ should be a small quantity consistent with the large value of $\xi$.

For a given value of $J^{\prime} / J$ between 0 and 2 we have varied $\gamma$ from 0 to 1 . In this interval of $\gamma$ we find regions with a massive behaviour of the gap described by (6) with $\xi$ smaller than the lattice size, together with small windows where the scaling is better described by (7) with $\Delta_{\infty}$ varying between 0.04 and 0.07 . The critical point is associated with the minimum in this region. The latter points are depicted in figure 3 and we associate them with the critical line conjectured in ref [11]. Strictely speaking, using the Lanczos method with $L$ up to 12 , we cannot rule out a very small gap in the region we believe is critical. However we think that this possibility is remote ( see ref [15] for similar difficulties in detecting gapless phases for the two leg ladders with ferromagnetic interchain couplings).

In figure 3 we have plotted our numerical results together with the continuous curve given by $J^{\prime} / J=2 \gamma^{2 / 3}$ which gives a reasonable overall fit. 
We can motivate this curve as follows. A dimerized spin $1 / 2$ chain has a gap which behaves as $J \gamma^{2 / 3}$ according to the Cross and Fisher law. This law can be proved using Conformal Field Theory. Indeed a dimerized spin 1/2 chain is described by a $S U(2)$ WZW model perturbed by a relevant operator with conformal dimension $1 / 2$, which by the way is the unique relevant operator available in the model [16]. On the other hand a uniform spin $1 / 2$ ladder, in the weak coupling limit $J / J^{\prime}<<1$, is described by two WZW models, corresponding to every chain, which are coupled through a relevant operator with dimension 1 [17]. This leads to a linear dependence on $J^{\prime}$ of the spin gap of uniform chains. The critical curve that we propose, i.e. $J^{\prime} / J=2 \gamma^{2 / 3}$, is an interesting combination between these two laws, which could be called the Cross-Fisher-ladder (CFL) law. From the previous conformal field theory arguments we see that the mass scale induced by both $J^{\prime}$ and $\gamma$ couplings behave as mass $\sim J \gamma^{2 / 3} \sim J^{\prime}$ and since the model is critical these two quantities should be identical. The proportionaly factor 2 in the $C F L$ law is fixed by the fact that at $\gamma=1$ the dimerized ladder becomes a chain with the shape of a "snake" (see figure 1b) [1], which is critical for $J^{\prime} / J=2$. Thus the two mechanisms for generating a spin gap cancel each other.

The critical curve of figure 3 separates two phases which can be neatly identified with the resonating valence bond phase of ladders (RVB) and the dimer phase of spin chains. We can try to understand why there should be a critical line between these two different regimes. On the RVB region the basic mechanism which lowers the energy is the resonance between parallel bonds on both rungs and legs which is proportional to $J^{\prime}$. In the dimer phase the bonds are fixed to staggered configurations and their energy is proportional to $J \gamma$. When both $J^{\prime}$ and $\gamma$ are non vanishing there is a competition between these two types of configurations: resonance is favored by $J^{\prime}$ but disfavored by $\gamma$, while staggering is favored by $\gamma$ but disfavored by $J^{\prime}$. The critical behaviour appears when there is a perfect balance between the two phenomena.

It may seem that the RVB and dimer phases of the alternating ladder are radically different. However, as shown in [15,[19], one can connect the dimer and the RVB phases with the Haldane phase of a spin 1 chain. This is not inconsistent with our results. All that says 
is that the path that connects the RVB and dimer phases of the dimerized 2-leg ladder lies outside our two dimensional phase diagram.

In summary, we have addressed the problem of whether the dimerized ground state survives the quantum interchain and alternating staggering fluctuations. We have found that the Lanczos's numerical results confirm the existence of a critical phase where the 2leg ladder remains gapless. This justifies that the "snake mechanism" presented before is also valid away of the strong-coupling/strong-staggering regime (the upper-right part of the phase diagram.)

Note added: After completion of this paper we have been aware of the paper of Flocke [20] which agrees essentially with our results. However a theoretical explanation of the phase diagram as well as the law $\gamma^{2 / 3}$ for the critical curve separating the dimer and the RVB phases are lacking in [20].

Acknowledgements: We would like to thank R. Shankar for discussions in the early stages of this work. We are also grateful to H. Nishimori for the package TITPACK version 2 which we have used for the diagonalization of spin- $1 / 2$ systems.

MAMD and GS acknowledges support from the DIGICYT under contract No. PB96/0906. JD acknowledges support from the DIGICYT under contract No. PB95/0123 


\section{FIGURE CAPTIONS}

Fig.1 a) A 2-leg ladder with alternating dimerized couplings $J(1 \pm \gamma)$ along the horizontal legs and $J^{\prime}$ along the vertical rungs. b) A maximally dimerized ladder with $\gamma=1$. c) A uniform 1D-Heisenberg chain emerging from the "snake" pattern when $\gamma=1$ and $J^{\prime} / J=2$.

Fig.2 A 3D-plot of the surface of gaps $\Delta\left(\gamma, J^{\prime} / J\right)_{L}$, for $L=10$, in the two-dimensional parameter space of couplings showing the existence of a steep valley which we associate to the critical curve in the phase diagram.

Fig.3 Phase diagram resulting from the analysis of the Lanczos results. The circles denote the extrapolated numerical results. The solid curve is the function $2 \gamma^{2 / 3}$ (explained in the text) which fits those circles quite well. RVB and Dimer phases are separated by this line. The dashed line corresponds to the perturbation result given in eq.(3), while the dotted-dashed line corresponds to the NLSM result given in eq.(5). 


\section{REFERENCES}

[1] E. Dagotto and T. M. Rice, Science 271 (1996) 618; see also "Search and Discovery," Physics Today, October, 1996.

[2] E. Dagotto, J. Riera, and D. J. Scalapino, Phys. Rev. B 45 (1992) 5744; M. Sigrist, T. M. Rice, and F. C. Zhang, Phys. Rev. B 49 (1994) 12058; S. R. White, R. M. Noack, and D. J. Scalapino, Phys. Rev. Lett. 73 (1994) 886; D. Poilblanc, H. Tsunetsugu, and T. M. Rice, Phys. Rev. B 50 (1994) 6511; H. Tsunetsugu, M. Troyer, and T. M. Rice, Phys. Rev. B 49 (1994) 16078.

[3] M. Uehara , T. Nagata, J. Akimitsu, H. Takahashi, N. Mori and K. Kinoshita, J. of Phys. Jpn, 65,2764 (1997).

[4] E. Manousakis, Rev. Mod. Phys. 63 (1991) 1.

[5] T. Barnes, E. Dagotto, J. Riera, and E. S. Swanson, Phys. Rev. B 47 (1993) 3196.

[6] M. Greven, R. J. Birgeneau, and U. J. Wiese, Phys. Rev. Lett. 77 (1996) 1865.

[7] S. R. White, Phys. Rev. Lett. 69, 2863 (1992). Phys. Rev. B 48, 10345 (1993).

[8] G. Sierra, M.A. Martín-Delgado, Phys. Rev. B 56, 8774 (1997).

G. Sierra, M. A. Martín-Delgado, J. Dukelsky, S. R. White, and D. J. Scalapino: condmat/9707335 ( to appear in PRB). J. Dukelsky, M. A. Martín-Delgado, T. Nishino, and G. Sierra: cond-mat/9710310. J. M. Roman, G. Sierra, J. Dukelsky, and M. A. MartínDelgado: cond-mat/9802150.

[9] J. K. Cullum and R. A. Willoughby, Lanczos Algorithms for Large Symmetric Eigenvalue Computations, Progress in Scientific Computing, Vol. 3 (Birkhäuser, Boston, 1985).

[10] E. Dagotto, Rev. Mod. Phys. 66, 763 (1994).

[11] M.A. Martín-Delgado, R. Shankar and G. Sierra, Phys. Rev. Lett. 77, 3443 (1996). 
[12] K. Totsuka and M. Suzuki, J. of Phys. Condens. Matter, 7,6079 (1995).

[13] M. C. Cross and D. S. Fisher, Phys. Rev. B 19, 402, (1979)..

[14] F. D. Haldane, Phys. Rev. Lett. 50, 1153 (1983); Phys.Lett. 93A, 464 (1983).

[15] K. Hida, J. Phys. Soc. Jpn. vol.60, 1347 (1991) .

[16] I. Affleck, Les Houches Lecture Notes, in : Fields, Strings and Critical Phenomena, ed. E. Brezin and J. Zinn-Justin (North-Holland, Amsterdam, 1988).

[17] H.J. Schulz, Phys. Rev. B 34, 6372 (1986); and cond-mat/9605075.

S.P. Strong and A.J. Millis, Phys. Rev. Lett. 69, 2419 (1992).

D.G. Shelton, A.A. Nersesyan and A.M. Tsvelik, Phys. Rev. B 53, 8521 (1996).

[18] K. Hida, Phys. Rev. B 45, 2207 (1992).

[19] S. R. White, Phys. Rev. B 53, 52 (1996).

[20] N. Flocke, Phys. Rev. B 56, 13673 (1997). 

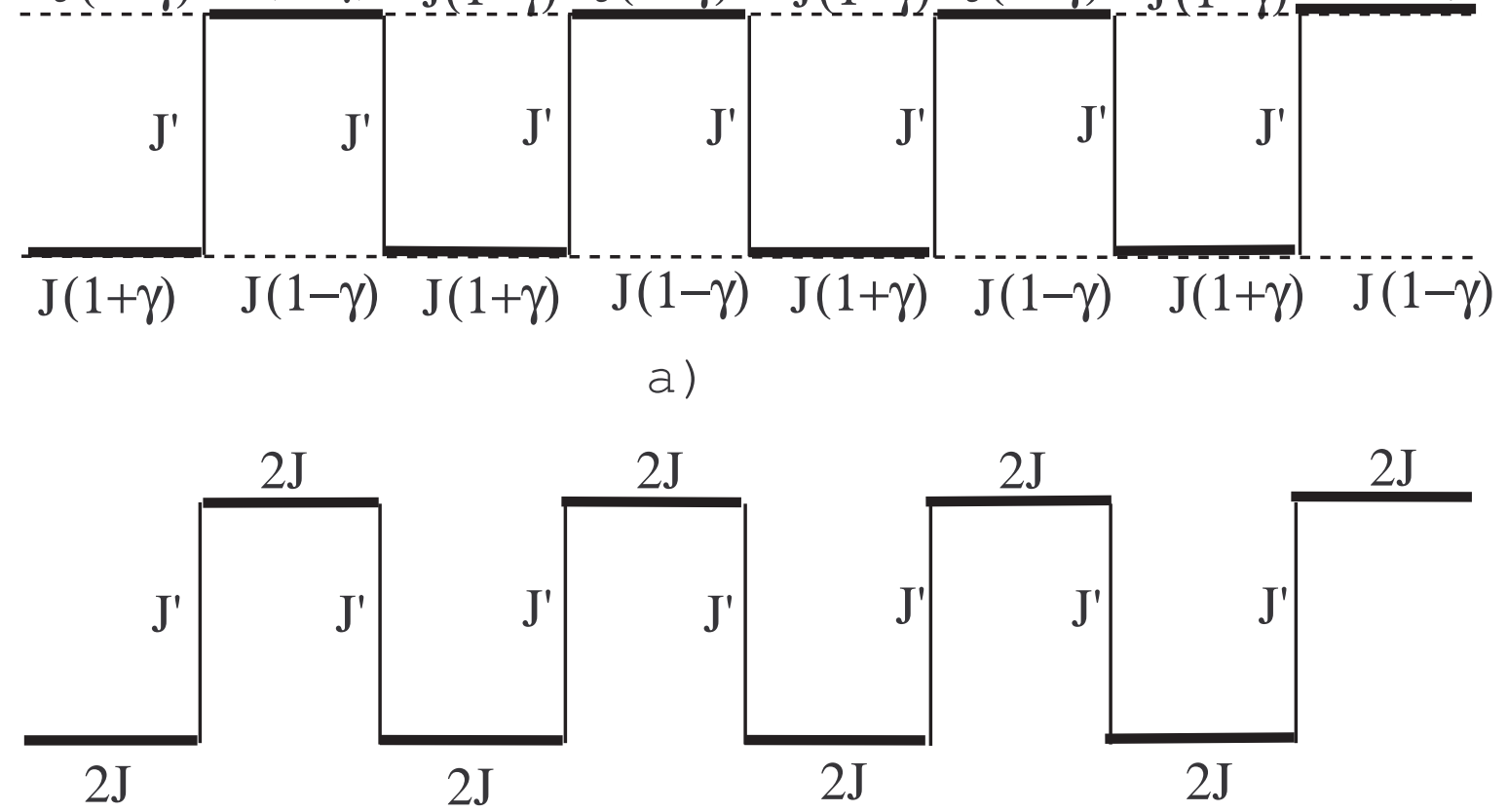

b)

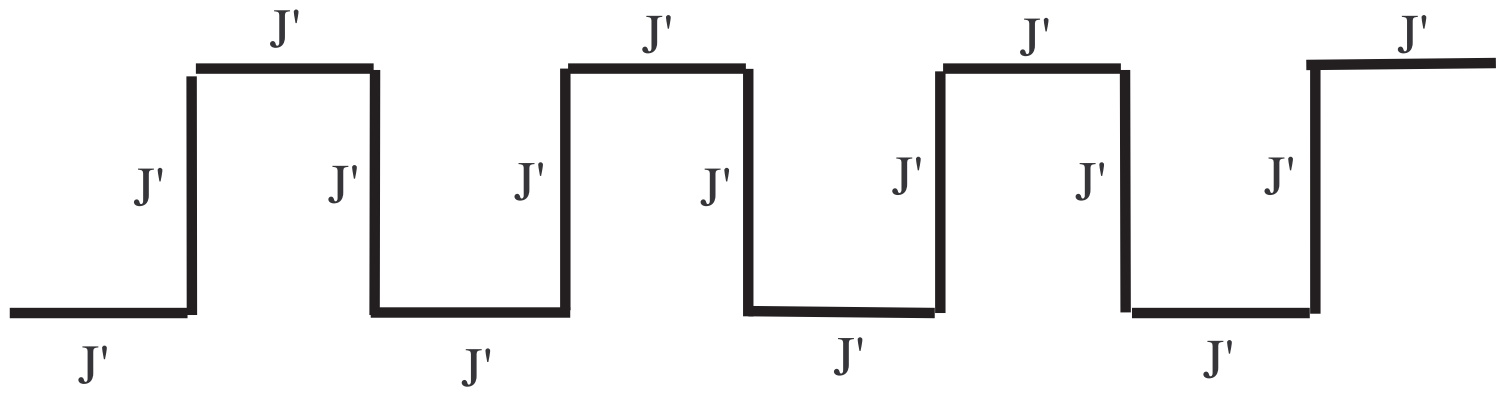

C)

Figure 1 


\section{Figure 2}

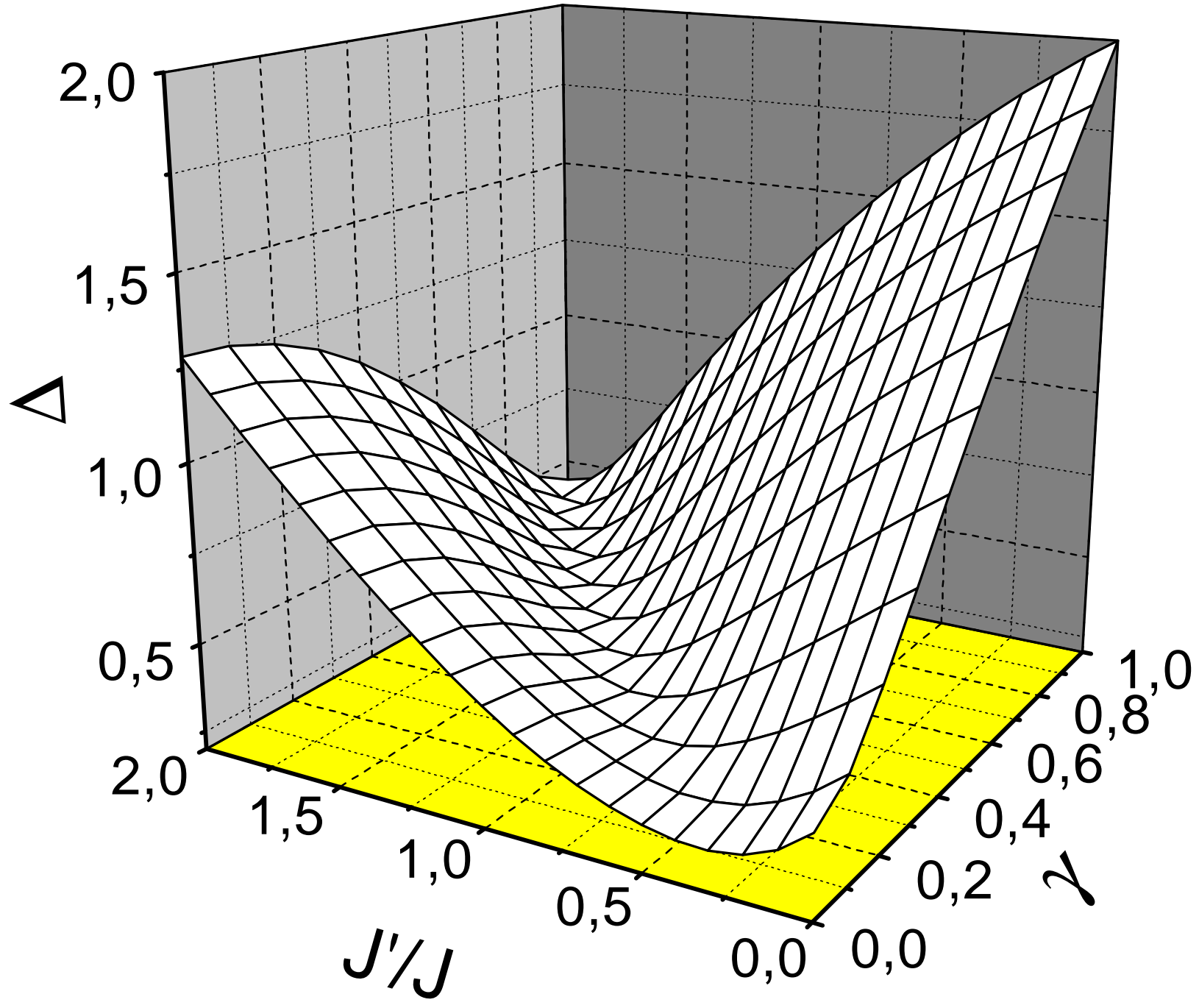


Figure 3

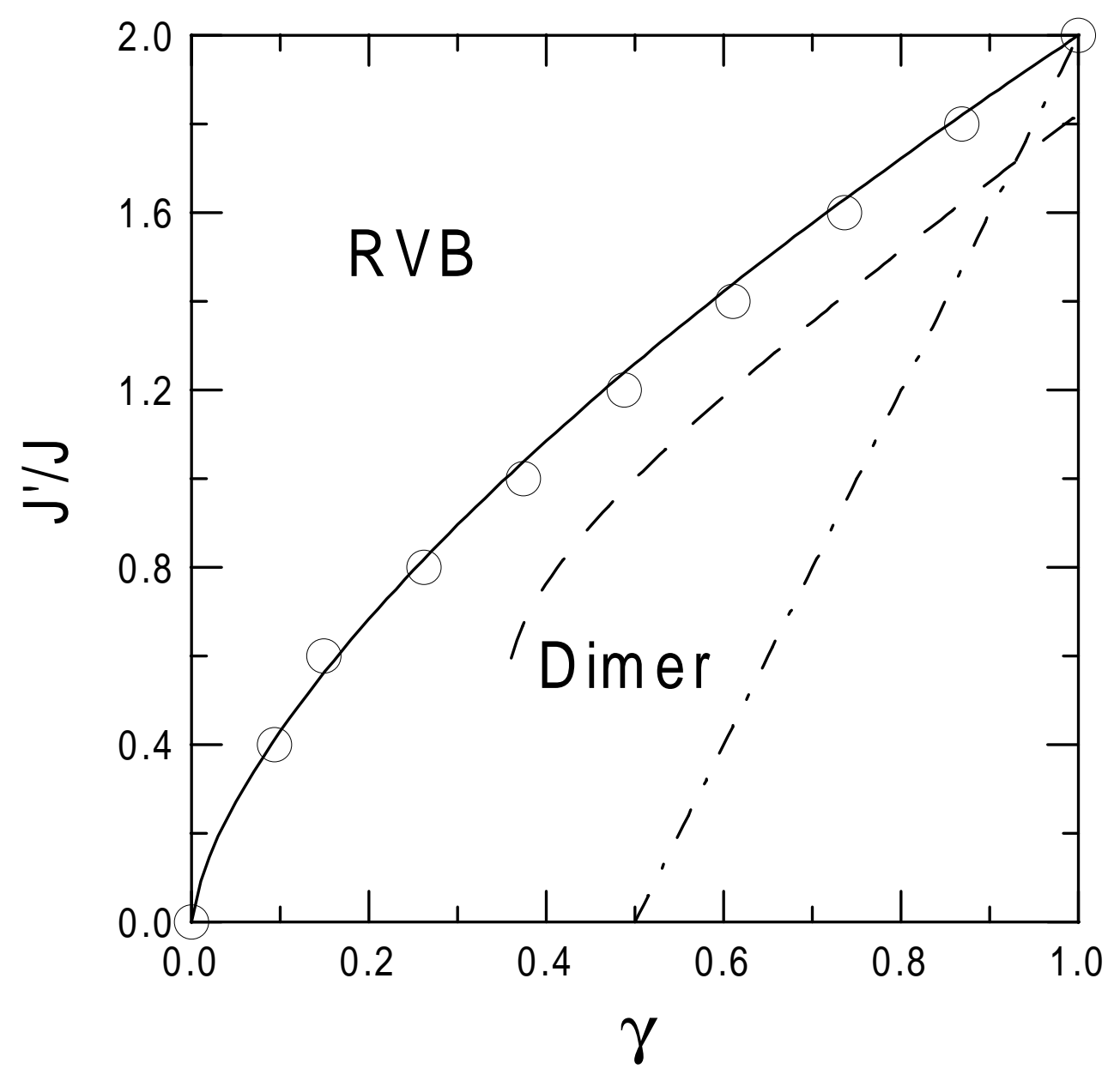

